also assayed the thrombin clotting time as a measure of blood coagulation, the euglobulin lysis time as a measure of fibrinolysis, and the inhibition of plasminogen activation by urokinase (an enzyme preparation which stimulates fibrinolysis) and by the blood platelets. The increase during pregnancy of both fibrinogen and plasminogen was highly significant. At term, allowing for the change in the volume of the plasma during pregnancy, the absolute amount of both enzyme precursors had doubled. The concentration of fibrinogen increased steadily throughout pregnancy, and the concentration was significantly correlated with the period of gestation. The concentration of plasminogen varied less during gestation, and increased slightly during labour.

There was no significant alteration in thrombin time. The mean platelet count was slightly reduced during pregnancy and lowest in labour but rose significantly in the first week after delivery. The most striking finding, however, was a steep increase in euglobulin lysis time, particularly towards the end of pregnancy. Statistical analysis showed that there was a significant. negative correlation between fibrinolysis and the gestation period. There was no evidence of the presence of inhibitors of plasminogen activation by urokinase and so this increase in fibrinolysis seemed to be the result of a decrease in the concentration of plasminogenactivating materials in the plasma. This decrease in. the ability to lyse fibrin while the coagulation of the blood remains normal probably maintains the placental circulation and prevents excessive blood loss, but might be the cause of some complications of pregnancy and of the increased frequency of and mortality from cerebral strokes in pregnant women.

A few weeks earlier the same group reported another investigation which showed that the concentration of immunologically detected fibrin degradation products did not change during pregnancy (Brit. Med. J., 3, 137; 1969). There was, however, a highly significant increase in these products of fibrinolysis during labour and again one week after delivery. There was an even greater increase after caesarean section, intra-uterine death or post-partum haemorrhage and in patients with abruptio placentae or eclampsia. They conclude that fibrinolysis can proceed locally at least in some tissues even though the general ability of the plasma to lyse fibrin is reduced.

\section{MENTAL DISEASE}

\section{Biochemical Causes}

A RECENT report from the World Health Organization establishes a strong case for linking much mental disease with malnutrition, particularly during very early life (Biochemistry of Mental Disorders, WHO Tech. Rep. Series No. 427, HMSO, 6s). An obvious example is iodine deficiency. Since the introduction of iodized salt in the high Alps, the endemic cretinism which used to horrify Victorian mountaineers has completely disappeared. The brain scems to stand up quite well to simple calorie deficiency; the loss in brain weight as a result of undernutrition is very small compared with the loss in body weight. Protein deficiency is much more serious: changes in the nerve cells of experimental animals include chromatolysis, foaming of the cytoplasm, degeneration of Nissl structures, neuronal loss and fibrous gliosis. Vitamin deficiency is also very serious. Vitamin A deficiency is primarily responsible for the high incidence of blindness in India. Thiamine deficiency causes a decrease in the cerebral respiration rate and in the level of acetyl choline. Riboflavin deficiency is associated with myelin degeneration and $\mathrm{B}_{12}$ deficiency with changes in the spinal cord. Probably every vitamin has a role in brain metabolism.

Mania and depression can be treated successfully, but are far from understood. These moods seem to be related to the metabolism of the cerebral monoamines, and monoamine oxidase inhibitors are important antidepressant drugs. Current work includes the analysis of body fluids during mania and depression and the clinical effects of feeding amine precursors. Curiously, the report makes no mention of L-dopa (Nature, 222, $889 ; 1969)$. Mania and depression are also associated with changes in water balance and sodium concentrations. Lithium is a useful treatment for these disorders and could be a good reagent for investigating the biochemistry of the processes involved.

All the work done so far on schizophrenia has achieved very little. The difficulty is that the voices and visions of schizophrenic patients have no known specific biochemical correlate. The only rational approach for the biochemist may be through the study of conditions that have broadly similar symptoms, such as the drug-induced psychoses. These have often been considered as model symptoms, and much more progress has now been made. Psychoses induced by either a single large dose of LSD or repeated doses of amphetamine are accompanied by similar metabolic changesincrease in serotonin and decrease in norepinephrine. In the brains of cats treated with amphetamine, the enzymes responsible for the metabolism of these amines have been mapped, and a decrease in monoamine oxidase activity has been observed in the amygdala. Inhibition of protein synthesis could be responsible for this decrease.

The mechanism of addiction to drugs is not settled. Some investigators have shown that tolerance to morphine is maintained in the rat for up to a year after a single injection. This suggests an immune response, and tolerance experiments are now being carried out in the presence of inhibitors of protein synthesis.

The WHO report ends with an evaluation of alternative approaches to research. Clinical studies are most usefully carried out on patients whose psychoses are periodic, so that they can act as their own controls. Averaging experiments are not very helpful because of individual differences between patients. Experiments with groups of animals can reveal relationships between experience and subsequent drug response. Biochemical studies are to some extent restricted by the heterogeneity of the tissue involved: it is not possible, for example, to dissect out the neurones from the glial cells, except in very small quantities. Nevertheless an impressive amount is known about the basic metabolism of the brain, including those processes that occur in rhythmic cycles, such as endocrine control and sleep.

\section{MARINE BIOLOGY}

\section{Prawns in Culture}

There are signs that the size of the prawn harvest will in future depend less on the coldness of the winter. 